Rabaska

Revue d'ethnologie de l'Amérique française

\title{
Edmond-Joseph Massicotte : revu et corrigé par David Karel
}

\section{Bernard Genest}

Volume 4, 2006

URI : https://id.erudit.org/iderudit/201771ar

DOI : https://doi.org/10.7202/201771ar

Aller au sommaire du numéro

Éditeur(s)

Société québécoise d'ethnologie

ISSN

1703-7433 (imprimé)

1916-7350 (numérique)

Découvrir la revue

Citer cette note

Genest, B. (2006). Edmond-Joseph Massicotte : revu et corrigé par David Karel. Rabaska, 4, 131-135. https://doi.org/10.7202/201771ar d'utilisation que vous pouvez consulter en ligne.

https://apropos.erudit.org/fr/usagers/politique-dutilisation/ 


\section{Edmond-Joseph Massicotte : revu et corrigé par David Karel}

Bernard Genest

Québec

Le 24 novembre 2005, le Musée national des beaux-arts du Québec inaugurait une exposition portant sur l'illustrateur québécois Edmond-Joseph Massicotte ${ }^{1}$. Préparée sous la direction du professeur David Karel, historien d'art de l'Université Laval, cette exposition était accompagnée d'une publication $^{2}$. Certains se souviendront peut-être qu'il y a une trentaine d'années, j'avais moi-même préparé un ouvrage sur Massicotte paru aux éditions du Boréal Express ${ }^{3}$. C'est dans le cadre de la préparation d'un mémoire de maîtrise en arts et traditions populaires que je m'étais intéressé à l'œuvre de Massicotte qui, en dépit de sa renommée, n'avait encore fait l'objet d'aucune étude approfondie. J'avais remarqué la place particulière qu'occupait ce dernier parmi les illustrateurs de son époque. Ses contemporains, en effet, le considéraient comme l'un des meilleurs « artistes canadiens » comme en témoigne l'auteur anonyme d'une notice nécrologique parue dans La Presse du 2 mars 1929.

Dans la préface de l'ouvrage, le directeur général du Musée national des beaux-arts du Québec, John R. Porter, rappelle l'orientation donnée au projet d'exposition : montrer au public québécois que Massicotte ne s'est pas cantonné dans l'exécution de scènes traditionnelles mais qu'il a été « aussi bien attiré par les sirènes de la modernité que soucieux de sauvegarder la mémoire du passé » (p. 11). Selon lui, cette ambivalence était le reflet d'une « société en évolution partagée entre passé et avenir, mais qui ne veut renoncer ni à l'un ni à l'autre ». D'où l'idée de faire reposer le concept sur la dualité tradition/modernité, non pas dans le but d'opposer ces éléments contradictoires mais plutôt de les faire se rencontrer pour expliquer la double personnalité

1. L'exposition Edmond-Joseph Massicotte, illustrateur, a été préparée par le Musée national des beaux-arts du Québec en collaboration avec l'Université Laval. Elle a été présentée au musée jusqu'au 13 août 2006.

2. David Karel, Edmond-Joseph Massicotte illustrateur, Québec, Musée national des beaux-arts du Québec et Les Presses de l'Université Laval, 2005, 222 p. ISBN 2-7637-8275-2

3. Bernard Genest, Massicotte et son temps, Montréal, Boréal Express, 1979, 240 p. ISBN 2-89052000-5. 
de l'artiste comme le précise David Karel en introduction : « la stratégie de l'exposition [...] se résume à confronter les deux sensibilités de Massicottela moderniste et la passéiste - afin que l'accomplissement de l'artiste puisse apparaître dans sa totalité » (p. 14). Du moins était-ce l'intention initiale. Le visiteur qui pénètre dans la salle d'exposition n'est donc pas surpris de voir que celle-ci se partage en deux parties. Un premier volet regroupe des œuvres autour des thèmes suivants : " Le Milieu », " La Belle Époque », "L'Art nouveau ». Ce volet s'intitule « Demain » dans la publication. Un deuxième volet, regroupe les œuvres consacrées à la représentation des traditions populaires. Les thèmes traités sont intitulés : "L'Invention de l'habitant », "Le Triomphe de la tradition », "Se souvenir ». Ce volet correspond à l'« Hier » du livre.

J'ai vu l'exposition, deux fois plutôt qu'une. Une première fois avant de lire le livre, une deuxième fois après sa lecture. Je dois reconnaître que j'ai été séduit par la sobriété de la présentation, le choix des œuvres et leur mise en contexte au moyen de vignettes concises et éclairantes. Des bornes interactives donnent accès à une documentation complémentaire des plus pertinentes. Le Musée national des beaux-arts du Québec nous a habitués à un standard de qualité qui fait école. L'exposition est une réussite sur le plan de la présentation et la publication est à l'égal de l'exposition. De belle facture, le livre est abondamment illustré et le texte, sobre mais dense, est soutenu par de nombreuses références et notes explicatives. Il faut souligner l'approche éclatée de David Karel dont l'expérience et l'érudition ont permis de contextualiser l'œuvre de Massicotte par rapport à son milieu et par rapport aux influences étrangères qui l'ont marqué. C'est davantage, bien sûr, dans le livre qu'on réalise l'ampleur de la recherche et son caractère multidisciplinaire. Le texte permet de développer en profondeur chacune des facettes de l'œuvre, ce qui est rarement possible dans le cadre d'une exposition. L'équipe d'experts du Musée n'a rien négligé pour rendre la matière accessible et conviviale. L'exposition et le livre qui l'accompagne vont certainement contribuer à faire redécouvrir aux Québécois l'éclectisme d'un artiste qui a profondément marqué l'imaginaire collectif.

Cependant, s'il importait de présenter au public le « jeune pionnier de l'avant-garde, ambitieux et novateur » qu'a été Massicotte, fallait-il vraiment accorder le même traitement aux travaux de jeunesse qu'à l'œuvre de maturité ? Aussi riche et intéressante que soit la production de ces années (de 1892 à 1909 environ), cette production est celle d'un artiste qui se cherche et qui n'a pas encore trouvé sa voie. Elle correspond à une période d'apprentissage faite d'une succession d'essais - certains réussis, d'autres moins - dans des domaines aussi variés que le portrait, l'illustration de contes et de nouvelles, le dessin journalistique, le dessin humoristique, la publicité et le 
style Art nouveau. La production de Massicotte à cette époque s'inspire de plusieurs illustrateurs connus mondialement. Elle est intéressante mais demeure secondaire par rapport à la production globale de l'artiste qui s'est surtout fait connaître comme illustrateur des traditions populaires. Car il faut bien admettre que c'est en effet l'œuvre «folklorique » qui a marqué l'imaginaire de plusieurs générations de Québécois et qui l'a rendu célèbre. C'est dans cette partie de son œuvre que Massicotte se libère des influences étrangères et trouve sa vraie personnalité, un genre, un style, une manière qui le caractérise au point que la signature, toujours présente, devient presque superflue. Comme pour justifier l'importance qu'il accorde aux travaux de jeunesse, David Karel parle d'une « vocation tardive d'illustrateur du terroir " (p. 163). Loin d'être tardive, cette vocation est l'aboutissement d'un long processus d'apprentissage qui le conduira à consacrer plus de vingt ans de sa carrière (de 1909 à son décès en 1929) à la réalisation d'un vaste catalogue illustré des us et coutumes des Québécois en milieu rural. Dès le moment où il met de l'avant ce projet, il abandonne à toutes fins pratiques les autres genres pour se consacrer presque exclusivement à l'œuvre folklorique, n'acceptant de commandes que dans la mesure où celles-ci ne l'éloignent pas de son champ d'intérêt.

Dans son introduction, Karel annonce qu'en deuxième partie du livre (qui suit le même plan que l'exposition), il va examiner « le passéisme d'un Massicotte tombé sous l'influence de la nouvelle science de la culture, l'ethnographie » (p. 15). On comprend, bien sûr, que l'auteur fait ici référence aux débuts de l'ethnologie québécoise. Il est vrai qu'en entreprenant de dresser l'inventaire illustré de pratiques traditionnelles, Edmond-Joseph Massicotte s'engageait dans une démarche identique à celle de ses amis folkloristes (parmi lesquels Édouard-Zotique, son frère, occupe une place importante) qui, pressentant la perte imminente d'un héritage commun, s'étaient donnés pour mission de recueillir les manifestations d'une culture dont ils pensaient être les derniers témoins. L'avenir allait démontrer que les traditions peuvent résister longtemps aux assauts répétés de la modernité et que les cultures sont moins fragiles qu'on ne le pense généralement. Aux yeux des folkloristes de cette époque toutefois, il était urgent d'inventorier le présent avant que le progrès ne transforme trop radicalement le mode de vie des ruraux. Dans le premier quart du vingtième siècle, la Révolution industrielle qui a transformé les paysages urbains n'a pas encore atteint les campagnes. L'agriculture se pratique selon les méthodes traditionnelles et la vie quotidienne, ponctuée par les fêtes religieuses, se déroule au rythme lent des saisons. Nous sommes ici dans un temps long qui s'inscrit en continuité avec le passé mais qui est bien réel. À ce propos Karel trouve paradoxal (p. 185) que mon livre s'intitule «Massicotte et son temps » alors que je ne me serais justement pas intéressé 
à « son » temps, c'est-à-dire à décrire l'époque qui était la sienne. On comprend que pour Karel l'univers représenté par Massicotte ne lui est pas contemporain mais qu'il s'agit d'un temps révolu. Mais de quel temps parle-t-on ici ? Du temps long de la campagne ou du temps en accéléré de la ville ? Karel reconnaît pourtant la valeur documentaire des dessins de Massicotte qui " épousait totalement l'attitude ethnographique de son frère " (p. 134). D'ailleurs les cahiers de croquis témoignent des « terrains » effectués par l'artiste dans le but de documenter in vivo les manières de faire et de vivre des habitants à la campagne. Que la vision de l'artiste soit empreinte d'un sentiment de nostalgie par rapport à une société qu'il idéalise et qu'il croit menacée par la modernité, cela est certain. Mais cette vision n'appartient pas au passé pour autant. Elle n'évoque pas un passé mais un présent en « son temps " encore observable. C'est celle d'un homme lucide qui s'est donné pour tâche de dresser une espèce de catalogue de pratiques, à ses yeux menacées. Doit-on le taxer de « passéiste » pour autant ? Ne fait-il pas, au contraire, figure de pionnier en souscrivant à cette nouvelle idéologie de la conservation du patrimoine culturel ? Car c'est bien de cela qu'il s'agit, de garder mémoire d'un héritage commun, une préoccupation toute récente dans le milieu qui l'entoure.

On sait que Massicotte n'ajoutait pas de couleur à ses dessins. Pourquoi alors avoir placé dans l'un des présentoirs de l'exposition des tubes de couleur et des pastilles de gouache ? Pourquoi, dans la publication, avoir fait une place si importante aux illustrations rehaussées à l'aquarelle ? La couverture, la quatrième, de même que seize pages centrales du livre, présentent en effet des images en couleur. Bien sûr, la couleur est attrayante mais elle suggère l'usage d'un procédé auquel l'artiste n'avait personnellement pas recours, préférant de beaucoup travailler en noir et blanc. Ce n'est pas d'aujourd'hui que circulent des images polychromes de l'artiste. Je me souviens que cela m'avait posé problème au moment de la publication de mes recherches jusqu'à ce que sa propre fille, madame Cécile Pothier, me confirme que ce n'était pas lui qui ajoutait la couleur à ses dessins ${ }^{4}$. Par ailleurs, commentant les cahiers de croquis (p. 30) faisant partie de la collection du Musée, Karel " observe le passage, sur les nus, d'une main pudique qui a artistiquement escamoté le dessin des organes génitaux ». Par endroits, nous apprend-il, c'est la « partie coupable » de la feuille qui est supprimée, dans d'autres cas, ce serait la page entière. L'auteur en conclut que « c'est le dessinateur lui-

4. Le seul dessin qu'il aurait lui-même rehaussé à la gouache serait cette composition destinée à la page couverture du Monde illustré du 28 décembre 1901, intitulée Retour de la messe de Minuit. D'ailleurs la vignette d'exposition nous apprend que c'est sur l'insistance des siens qu'il aurait ajouté de la couleur au dessin. 
même qui accomplit ce rituel victorien, en songeant, peut-être, à la curiosité de son enfant ». Il ajoute y voir une trace de repentir qui " grandit au fur et à mesure que l'on examine ces cahiers ». Je puis affirmer que ce n'est pas l'artiste qui a posé ce geste. Je tiens l'information de source sûre, c'est-àdire de la personne même qui a manié les ciseaux ${ }^{5}$.

On ne peut que féliciter le directeur général du Musée national des beauxarts du Québec d'avoir sorti de l'ombre Edmond-Joseph Massicotte pour le faire entrer de plain-pied dans l'histoire de l'art québécois. Ce n'est toutefois pas d'aujourd'hui que le Musée compte des cuvres de cet artiste dans ses collections. En fait, le Musée détient une grande partie de ses œuvres. Certaines ont été cédées du vivant de l'artiste. D'autres ont été acquises en 1932, mais la majorité provient d'un achat réalisé en 1976. On peut s'étonner que le Musée ait attendu si longtemps pour les présenter au public dans le cadre d'une exposition qui soit entièrement consacrée à leur auteur. Fallait-il absolument, pour que Massicotte puisse entrer par la grande porte au Musée, le présenter sous un jour nouveau ? L'artiste, déjà connu et admiré du public, ne pouvait-il mériter cet hommage pour ce qu'il est et demeure avant tout : l'illustrateur de « nos » traditions populaires ? On doit aussi féliciter David Karel pour avoir approfondi la connaissance de l'œuvre en la situant dans une perspective à la fois large et globale. Toutefois, en dépit de toutes les nuances apportées, on sent bien que c'est davantage le jeune dessinateur « de l'avant-garde, ambitieux et innovateur » qui intéresse l'historien d'art plutôt que « l'homme assagi qui se consacre à la sauvegarde des traditions ». Aussi, on est en droit de se demander si l'objectif de départ a vraiment été atteint. En opposant l'œuvre de jeunesse à l'œuvre de maturité, pouvait-on réellement espérer en arriver à « concilier les deux sensibilités » de l'artiste ?

5. On comprendra que, par discrétion, je ne donne pas le nom de cette personne dans le cadre de cet article ni les motifs qui l'ont amenée à poser ce geste. 